\title{
N,N-Dimethyl Glycine Promoted Ullmann Coupling Reaction of Phenols and Aryl Halides
}

\author{
Dawei Ma* and Qian Cai \\ State Key Laboratory of Bioorganic and Natural Products Chemistry, Shanghai \\ Institute of Organic Chemistry, Chinese Academy of Sciences, 354 Fenglin Lu, \\ Shanghai 200032, China.
}

Supporting Information 


\section{Experimental}

General Procedure for Table 1: A mixture of $2 \mathrm{mmol}$ of 4-iodoanisole, 3 mmol of phenol, $4 \mathrm{mmol}$ of $\mathrm{Cs}_{2} \mathrm{CO}_{3}, 0.04 \mathrm{mmol}$ of copper catalyst, $0.15 \mathrm{mmol}$ of the additive, and $4 \mathrm{~mL}$ of dioxane in a sealed tube was heated at $90{ }^{\circ} \mathrm{C}$ under nitrogen atmosphere for $22 \mathrm{~h}$. The cooled mixture was partitioned between ethyl acetate and water. The organic layer was separated, and the aqueous layer was extracted with ethyl acetate. The combined organic layers were washed with brine, dried over $\mathrm{Na}_{2} \mathrm{SO}_{4}$, and concentrated in vacuo. The residual oil was loaded on a silica gel column and eluted with ethyl acetate/petroleum ether (1/30) to afford the product.

General Procedure for Table 2: A mixture of $2 \mathrm{mmol}$ of aryl iodide, $3 \mathrm{mmol}$ of phenol, $4 \mathrm{mmol}$ of $\mathrm{Cs}_{2} \mathrm{CO}_{3}, 0.04 \mathrm{mmol}$ of copper catalyst, $0.15 \mathrm{mmol}$ of $N, N$-dimethylglycine hydrochlroide salt (or $0.2 \mathrm{mmols}$ of $\mathrm{CuI}$ and $0.6 \mathrm{mmols}$ of $\mathrm{N}, \mathrm{N}$-dimethylglycine hydrochlroide salt in some cases), and $4 \mathrm{~mL}$ of dioxane in a sealed tube was heated at $90{ }^{\circ} \mathrm{C}$ under nitrogen atmosphere. After the reaction completed monitored by TLC, the cooled mixture was partitioned between ethyl acetate and water. The organic layer was separated, and the aqueous layer was extracted with ethyl acetate. The combined organic layers were washed with brine, dried over $\mathrm{Na}_{2} \mathrm{SO}_{4}$, and concentrated in vacuo. The residual oil was loaded on a silica gel column and eluted with ethyl acetate/petroleum ether (1/30) to afford the product.

General Procedure for Table 3: A mixture of $2 \mathrm{mmol}$ of aryl bromide, 3 mmol of phenol, $4 \mathrm{mmol}$ of $\mathrm{Cs}_{2} \mathrm{CO}_{3}, 0.2 \mathrm{mmol}$ of copper catalyst, $0.6 \mathrm{mmol}$ of 
$N, N$-dimethylglycine hydrochlroide salt, and $4 \mathrm{~mL}$ of dioxane in a sealed tube was heated at $90{ }^{\circ} \mathrm{C}$ under nitrogen atmosphere. After the reaction completed monitored by TLC, the cooled mixture was partitioned between ethyl acetate and water. The organic layer was separated, and the aqueous layer was extracted with ethyl acetate. The combined organic layers were washed with brine, dried over $\mathrm{Na}_{2} \mathrm{SO}_{4}$, and concentrated in vacuo. The residual oil was loaded on a silica gel column and eluted with ethyl acetate/petroleum ether (1/30) to afford the product.

1-Methoxy-4-phenoxybenzene $\mathbf{1}^{1} .{ }^{1} \mathrm{H}$ NMR $\left(\mathrm{CDCl}_{3}, 300 \mathrm{MHz}\right) \delta 3.80(\mathrm{~s}$, 3H), 6.87-7.00 (m, 7 H), 7.29 (m, 1H); MS m/z $200\left(\mathrm{M}^{+}\right)$, 185, 169, 129, 123, 95, 77, $63,51,50,41$.

Phenyl 4-methylphenyl ether $2^{1} .{ }^{1} \mathrm{H} \mathrm{NMR}\left(\mathrm{CDCl}_{3}, 300 \mathrm{MHz}\right) \delta 2.34(\mathrm{~s}, 3 \mathrm{H})$, $6.92(\mathrm{~d}, J=8.1 \mathrm{~Hz}, 2 \mathrm{H}), 7.00(\mathrm{~d}, J=7.8 \mathrm{~Hz}, 2 \mathrm{H}), 7.07(\mathrm{~m}, 1 \mathrm{H}), 7.16(\mathrm{~m}, 2 \mathrm{H}), 7.32(\mathrm{~m}$, 2H); MS m/z $184\left(\mathrm{M}^{+}\right), 169,155,141,128,115,91,77,65,51,41$.

3, 5-Dimethylphenyl phenyl ether $3^{2} .{ }^{1} \mathrm{H} \mathrm{NMR}\left(\mathrm{CDCl}_{3}, 300 \mathrm{MHz}\right) \delta 2.28(\mathrm{~s}$, 6H), $6.64(\mathrm{~s}, 2 \mathrm{H}), 6.73(\mathrm{~s}, 1 \mathrm{H}), 7.01(\mathrm{~m}, 3 \mathrm{H}), 7.32(\mathrm{~m}, 2 \mathrm{H}) ; \mathrm{MS} \mathrm{m} / z 198\left(\mathrm{M}^{+}\right), 183$, $169,155,129,115,105,98,91,77,65,51,41$.

3-Nitrophenyl phenyl ether $4^{3} .{ }^{1} \mathrm{H}$ NMR $\left(\mathrm{CDCl}_{3}, 300 \mathrm{MHz}\right) \delta 7.07(\mathrm{~m}, 2 \mathrm{H})$, $7.22(\mathrm{~m}, 1 \mathrm{H}), 7.32(\mathrm{~m}, 1 \mathrm{H}), 7.40-7.53(\mathrm{~m}, 3 \mathrm{H}), 7.80(\mathrm{t}, J=2.1 \mathrm{~Hz}, 1 \mathrm{H}) ; \mathrm{MS} m / z 215$ $\left(\mathrm{M}^{+}\right), 198,168,141,128,115,102,92,77,63,51$.

1,4-Diphenoxybenzene. ${ }^{1} \mathrm{H} \mathrm{NMR}\left(\mathrm{CDCl}_{3}, 300 \mathrm{MHz}\right) \delta 7.02(\mathrm{~m}, 8 \mathrm{H}), 7.11(\mathrm{~m}$, 2H), $7.36(\mathrm{~m}, 4 \mathrm{H})$; MS m/z $262\left(\mathrm{M}^{+}\right), 233,185,168,157,141,129,115,103,91,77$, 63, 51 . 
Diphenyl ether $6{ }^{4} .{ }^{1} \mathrm{H}$ NMR $\left(\mathrm{CDCl}_{3}, 300 \mathrm{MHz}\right) \delta 6.97(\mathrm{~m}, 4 \mathrm{H}), 7.08(\mathrm{~m}, 2 \mathrm{H})$, 7.30 (m, 4H); MS m/z $170\left(\mathrm{M}^{+}\right), 155,141,128,115,94,77,65,51$.

1-Methoxy-2-phenoxybenzene $7^{5} .{ }^{1} \mathrm{H}$ NMR $\left(\mathrm{CDCl}_{3}, 300 \mathrm{MHz}\right) \delta 3.84(\mathrm{~s}$, 3H), $6.95(\mathrm{~m}, 6 \mathrm{H}), 7.30(\mathrm{t}, J=8.1 \mathrm{~Hz}, 2 \mathrm{H}) ; \mathrm{MS} m / z 200\left(\mathrm{M}^{+}\right), 185,169,129,123,95$, $77,63,51,50,41$.

1-Methoxy-4-m-tolyloxybenzene 8. ${ }^{1} \mathrm{H} \mathrm{NMR}\left(\mathrm{CDCl}_{3}, 300 \mathrm{MHz}\right) \delta 2.32$ (s, 3H), $3.81(\mathrm{~s}, 3 \mathrm{H}), 6.73(\mathrm{~d}, J=8.4 \mathrm{~Hz}, 2 \mathrm{H}), 6.87(\mathrm{~m}, 3 \mathrm{H}), 6.98(\mathrm{~m}, 2 \mathrm{H}), 7.21(\mathrm{t}, J=7.8$ $\mathrm{Hz}, 1 \mathrm{H}) ; \mathrm{MS} m / z 214\left(\mathrm{M}^{+}\right), 199,171,143,128,91,77,65,51,41$.

Benzyl (4-m-tolyoxyphenyl)amine $9^{6} .{ }^{1} \mathrm{H} \mathrm{NMR}\left(\mathrm{CDCl}_{3}, 300 \mathrm{MHz}\right) \delta 2.30(\mathrm{~s}$, $3 \mathrm{H}), 4.32(\mathrm{~s}, 2 \mathrm{H}), 6.62(\mathrm{~m}, 2 \mathrm{H}), 6.48(\mathrm{~m}, 2 \mathrm{H}), 6.74(\mathrm{~m}, 1 \mathrm{H}), 6.91(\mathrm{~m}, 2 \mathrm{H}), 7.15(\mathrm{~m}$, 1H), 7.35 (m, 5 H); MS m/z $289\left(\mathrm{M}^{+}\right), 212,198,180,168,155,143,128,115,91,77$, 65, 63, 51, 41; Anal. Calcd. for $\mathrm{C}_{20} \mathrm{H}_{19} \mathrm{NO}$ requires C: 83.01, $\mathrm{H}: 6.62, \mathrm{~N}: 4.84$, found C: 83.03, H: 6.33, N: 4.79 .

Phenyl $\boldsymbol{m}$-tolyl ether $10^{7} .{ }^{1} \mathrm{H}$ NMR $\left(\mathrm{CDCl}_{3}, 300 \mathrm{MHz}\right) \delta 2.32(\mathrm{~s}, 3 \mathrm{H}), 6.82(\mathrm{~m}$, 2H), $6.92(\mathrm{~m}, 1 \mathrm{H}), 7.01(\mathrm{~m}, 2 \mathrm{H}), 7.09(\mathrm{~m}, 1 \mathrm{H}), 7.21(\mathrm{~m}, 1 \mathrm{H}), 7.32(\mathrm{~m}, 2 \mathrm{H}) ; \mathrm{MS} \mathrm{m} / z$ $184\left(\mathrm{M}^{+}\right), 169,155,141,128,115,91,77,65,63,51,41$.

1-(4-tert-Butylphenoxy)-4-methoxybenzene 11. ${ }^{1} \mathrm{H} \mathrm{NMR}\left(\mathrm{CDCl}_{3}, 300 \mathrm{MHz}\right)$ $\delta 1.32(\mathrm{~s}, 9 \mathrm{H}), 3.82(\mathrm{~s}, 3 \mathrm{H}), 6.87(\mathrm{~m}, 4 \mathrm{H}), 7.98(\mathrm{~d}, J=9.3 \mathrm{~Hz}, 2 \mathrm{H}), 7.30(\mathrm{~d}, J=9.3 \mathrm{~Hz}$, 2H); MS m/z $256\left(\mathrm{M}^{+}\right), 241,226,213,198,181,165,129,123,95,77,65,51,41$.

1-(4-tert-Butylphenoxy)-4-fluorobenzene $12^{6} .{ }^{1} \mathrm{H} \mathrm{NMR}\left(\mathrm{CDCl}_{3}, 300 \mathrm{MHz}\right) \delta$ $1.35(\mathrm{~s}, 9 \mathrm{H}), 6.80(\mathrm{~m}, 3 \mathrm{H}), 6.98(\mathrm{~m}, 2 \mathrm{H}), 7.28(\mathrm{~m}, 1 \mathrm{H}), 7.38(\mathrm{~m}, 2 \mathrm{H}) ; \mathrm{MS} \mathrm{m} / \mathrm{z} 244$ $\left(\mathrm{M}^{+}\right), 229,201,118,117,100,91,77,65,51$; Anal. Calcd. for $\mathrm{C}_{16} \mathrm{H}_{17} \mathrm{FO}$ requires $\mathrm{C}$ : 
78.66, H: 7.01, found C: 78.81, H: 7.17.

1-[4-(Naphthalen-2-yloxy)phenyl]ethannone $\mathbf{1 3}^{6} \cdot{ }^{1} \mathrm{H}$ NMR $\left(\mathrm{CDCl}_{3}, 300\right.$ MHz) $\delta 2.61(\mathrm{~s}, 3 \mathrm{H}), 7.06(\mathrm{~m}, 2 \mathrm{H}), 7.26(\mathrm{~m}, 1 \mathrm{H}), 7.47(\mathrm{~m}, 3 \mathrm{H}), 7.78(\mathrm{~m}, 1 \mathrm{H}), 7.89(\mathrm{~m}$, 2H), 7,96 (m, 2H); MS m/z $262\left(\mathrm{M}^{+}\right), 247,218,189,127,115,101,95,83,77,63$, 51,43; Anal. Calcd. for $\mathrm{C}_{18} \mathrm{H}_{14} \mathrm{O}_{2}$ requires C: 82.42, H: 5.38, found C: 82.47, H: 5.58. 4-(2,4-Dimethylphenoxy)benzonitrile $14^{6} .{ }^{1} \mathrm{H}$ NMR $\left(\mathrm{CDCl}_{3}, 300 \mathrm{MHz}\right) \delta$ $2.11(\mathrm{~s}, 3 \mathrm{H}), 2.34(\mathrm{~s}, 3 \mathrm{H}), 6.87(\mathrm{~m}, 3 \mathrm{H}), 7.02(\mathrm{~m}, 1 \mathrm{H}), 7.09$ (s, 1H), $7.56(\mathrm{~m}, 2 \mathrm{H}) ; \mathrm{MS}$ $m / z 223\left(\mathrm{M}^{+}\right), 208,190,180,167,153,140,127,121,105,97,79,77,65,51,41$; Anal. Calcd. for $\mathrm{C}_{15} \mathrm{H}_{13} \mathrm{NO}$ requires $\mathrm{C}: 80.69, \mathrm{H}: 5.87, \mathrm{~N}: 6.27$, found $\mathrm{C}: 80.52, \mathrm{H}$ : $5.79, \mathrm{~N}: 6.15$.

1-(2-Methoxyphenoxy)-3,5-dimethylbenzene 15. ${ }^{1} \mathrm{H}$ NMR $\left(\mathrm{CDCl}_{3}, 300\right.$ MHz) $\delta 2.27(\mathrm{~s}, 6 \mathrm{H}), 3.85(\mathrm{~s}, 3 \mathrm{H}), 6.58(\mathrm{~s}, 2 \mathrm{H}), 6.69(\mathrm{~s}, 1 \mathrm{H}), 6.96(\mathrm{~m}, 3 \mathrm{H}), 7.11(\mathrm{~m}$, 1H); MS m/z $228\left(\mathrm{M}^{+}\right), 213,198,195,182,169,119,113,105,95,91,79,77,65,57$, 43. Anal. Calcd. for $\mathrm{C}_{15} \mathrm{H}_{16} \mathrm{O}_{2}$ requires C: 78.92, H: 7.06, found C: 78.74, H: 7.41.

1-Methoxy-2-p-tolyloxybenzene $16^{8} .{ }^{1} \mathrm{H} \mathrm{NMR}\left(\mathrm{CDCl}_{3}, 300 \mathrm{MHz}\right) \delta 2.34(\mathrm{~s}$, 3H), 3.88 (s, 3H), $6.90(\mathrm{~m}, 4 \mathrm{H}), 6.94(\mathrm{~m}, 1 \mathrm{H}), 7.13(\mathrm{~m}, 3 \mathrm{H})$; MS m/z $214\left(\mathrm{M}^{+}\right), 184$, $171,153,141,128,105,91,77,65,52,43$.

Biphenyl-4-yl $\boldsymbol{m}$-tolyl ether $\mathbf{1 7}^{8} .{ }^{1} \mathrm{H}$ NMR $\left(\mathrm{CDCl}_{3}, 300 \mathrm{MHz}\right) \delta 2.35(\mathrm{~s}, 3 \mathrm{H})$, $6.88(\mathrm{~m}, 2 \mathrm{H}), 6.93(\mathrm{~d}, J=7.5 \mathrm{~Hz}, 1 \mathrm{H}), 7.06(\mathrm{~m}, 2 \mathrm{H}), 7.23(\mathrm{~m}, 1 \mathrm{H}), 7.35(\mathrm{~m}, 1 \mathrm{H}), 7.43$ (m, 2H), $7.58(\mathrm{~m}, 4 \mathrm{H}) ; \mathrm{MS} \mathrm{m} / z 260\left(\mathrm{M}^{+}\right), 241,231,217,202,191,178,169,152,141$, $127,115,102,91,77,65,51$. 
4-Chlorophenyl $\boldsymbol{m}$-tolyl ether $1 \mathbf{1 8}^{9} .{ }^{1} \mathrm{H} \mathrm{NMR}\left(\mathrm{CDCl}_{3}, 300 \mathrm{MHz}\right) \delta 2.33(\mathrm{~s}$, 3H), $6.81(\mathrm{~m}, 2 \mathrm{H}), 6.93(\mathrm{~m}, 3 \mathrm{H}), 7.26(\mathrm{~m}, 3 \mathrm{H})$; MS m/z 220, 218, 203, 190, 183, 168, $155,125,108,91,76,65,63,51,41$.

2-(4-Methoxyphenoxy)naphthalene $19^{6} .{ }^{1} \mathrm{H} \mathrm{NMR}\left(\mathrm{CDCl}_{3}, 300 \mathrm{MHz}\right) \delta 3.81$ (s, 3H), $6.92(\mathrm{~d}, J=6.9 \mathrm{~Hz}, 2 \mathrm{H}), 7.03(\mathrm{~d}, J=7.9 \mathrm{~Hz}, 2 \mathrm{H}), 7.17(\mathrm{~m}, 1 \mathrm{H}), 7.23(\mathrm{~m}, 1 \mathrm{H})$, $7.39(\mathrm{~m}, 2 \mathrm{H}), 7.63(\mathrm{~d}, J=8.7 \mathrm{~Hz}, 1 \mathrm{H}), 7.78(\mathrm{~d}, J=8.7 \mathrm{~Hz}, 2 \mathrm{H}) ; \mathrm{MS} m / z 250\left(\mathrm{M}^{+}\right)$, 235, 222, 207, 189, 179, 127, 115, 101, 95, 89, 77, 63, 57, 51, 41; Anal. Calcd. for $\mathrm{C}_{17} \mathrm{H}_{14} \mathrm{O}_{2}$ requires C: 81.58, H: 5.64, found C: 81.30, H: 5.79.

Bis-(4-methoxyphenyl)ether $20^{10} .{ }^{1} \mathrm{H}$ NMR $\left(\mathrm{CDCl}_{3}, 300 \mathrm{MHz}\right) \delta 3.79(\mathrm{~s}, 6$ H), $6.83(\mathrm{~d}, J=9.3 \mathrm{~Hz}, 4 \mathrm{H}), 6.91(\mathrm{~d}, J=9.3 \mathrm{~Hz}, 4 \mathrm{H}) ; \mathrm{MS} m / z 230\left(\mathrm{M}^{+}\right), 215,200$, $187,172,155,144,123,115,107,95,92,77,64,53,41$.

1-(4-Chlorophenoxy)-4-methoxybenzene 21. ${ }^{1} \mathrm{H} \mathrm{NMR}\left(\mathrm{CDCl}_{3}, 300 \mathrm{MHz}\right) \delta$ $3.80(\mathrm{~s}, 3 \mathrm{H}), 6.89(\mathrm{~m}, 4 \mathrm{H}), 6.95(\mathrm{~m}, 2 \mathrm{H}), 7.23(\mathrm{~d}, J=9.0 \mathrm{~Hz}, 2 \mathrm{H}) ; \mathrm{MS} \mathrm{m} / z 236\left(\mathrm{M}^{+}\right)$ 234, 219, 191, 184, 171, 163, 156, 139, 128, 123, 111, 99, 92, 85, 75, 63, 57, 50, 41.

4 '-tert.-Butyl-4-nitro-diphenylether 22. ${ }^{1} \mathrm{H} \mathrm{NMR}\left(\mathrm{CDCl}_{3}, 300 \mathrm{MHz}\right) \delta 1.35$ (s, 9H), $6.99(\mathrm{~d}, J=2.7 \mathrm{~Hz}, 2 \mathrm{H}), 7.02(\mathrm{~d}, J=2.7 \mathrm{~Hz}, 2 \mathrm{H}), 7.42(\mathrm{~d}, J=9.3 \mathrm{~Hz}, 2 \mathrm{H})$, $8.18(\mathrm{~d}, J=9.3 \mathrm{~Hz}, 2 \mathrm{H}) ; \mathrm{MS} m / z 271\left(\mathrm{M}^{+}\right), 256,240,228,210,195,182,165,152$, $139,128,118,103,91,85,76,57,51,41$.

3-(4-Methoxyphenoxy)benzoic acid 23. ${ }^{1} \mathrm{H} \mathrm{NMR}\left(\mathrm{CDCl}_{3}, 300 \mathrm{MHz}\right) \delta 3.84$ (s, 3H), $6.92(\mathrm{~m}, 2 \mathrm{H}), 7.03(\mathrm{~m}, 2 \mathrm{H}), 7.22(\mathrm{~m}, 1 \mathrm{H}), 7.42(\mathrm{~m}, 1 \mathrm{H}), 7.64(\mathrm{~m}, 1 \mathrm{H}), 7.80$ (m, 1H); MS m/z $244\left(\mathrm{M}^{+}\right), 229,212,199,183,173,157,129,123,92,76,65,51,41$, Anal. Calcd. for $\mathrm{C}_{14} \mathrm{H}_{12} \mathrm{O}_{4}$ requires C: $68.85, \mathrm{H}: 4.95$, found C: 68.82, H: 4.83 . 
2,4-Dimethyl-1-p-tolyloxybenzene $24 .{ }^{1} \mathrm{H}$ NMR $\left(\mathrm{CDCl}_{3}, 300 \mathrm{MHz}\right) \delta 2.20(\mathrm{~s}$, 3H), 2.31 (br s, 6H), $6.78(\mathrm{~m}, 3 \mathrm{H}), 6.95$ (m, 1H), 7.06 (m, 3H); MS m/z $212\left(\mathrm{M}^{+}\right), 197$, 179, 169, 154, 120, 105, 91, 77, 65, 51, 41; Anal. Calcd. for $\mathrm{C}_{15} \mathrm{H}_{16} \mathrm{O}$ requires $\mathrm{C}$ : 84.87, H: 7.60, found C: 84.87, H: 7.79.

1-Methoxy-4-o-tolyloxybenzene $\mathbf{2 5}^{2} .{ }^{1} \mathrm{H} \mathrm{NMR}\left(\mathrm{CDCl}_{3}, 300 \mathrm{MHz}\right) \delta 2.28(\mathrm{~s}$, 3H), $3.79(\mathrm{~s}, 3 \mathrm{H}), 6.77(\mathrm{~m}, 5 \mathrm{H}), 7.00(\mathrm{~m}, 1 \mathrm{H}), 7.13(\mathrm{~m}, 1 \mathrm{H}), 7.22(\mathrm{~m}, 1 \mathrm{H}) ; \mathrm{MS} \mathrm{m} / z$ $214\left(\mathrm{M}^{+}\right), 199,181,171,153,143,123,108,91,78,65,51,41$.

\section{References}

1. Haga, N.; Takayanagi, H. J. Org. Chem. 1996, 61, 735-745.

2. Aranyos, A.; Old, D. W.; Kiyomori, A.; Wolfe, J. P.; Sadighi, J. P.; Buchwald, S. L. J. Amer. Chem. Soc. 1999, 121, 4369-4378.

3. Barton, D. H. R.; Yadav-Bhatnagar, N.; Finet, J.-P.; Khamsi, J.; Motherwell, W. B. Stanforth, S. P. Tetrahedron 1987, 43, 323-332.

4. Nevalainen, T.; Kohlemainen, E.; Saeaemaenen, M.-L.; Kauppinen, R. Magn. Reson. Chem. 1993, 100-104.

5. Carte, B.; Faulkner, D. J.; Tetrahedron 1981, 37, 2335-2340.

6. Bates, R. B.; Janda, K. D. J. Org. Chem. 1982, 47, 4374-4376.

7. Gujadhur, R.; Venkataraman, D. Synth. Commun. 2001, 2865-2880.

8. Renger, B. Phosphorus Sulfur 1988, 35, 215-218.

9. Wang, L.; Xi, H.; Sun, X.; Shen, Y.; Yang, Y.; Pan, Y.; Hu, H. Synth. Commun. 
2000, 227-234.

10. Pearson, A. J.; Gelormini, A. M. J. Org. Chem. 1994, 59, 4561-4570. 